DAMTP-06-24

hep-th/0604024

\title{
Self-gravitating Yang Monopoles in all Dimensions
}

\author{
G.W. Gibbons ${ }^{1}$ and P.K. Townsend ${ }^{2}$ \\ Department of Applied Mathematics and Theoretical Physics \\ Centre for Mathematical Sciences, University of Cambridge \\ Wilberforce Road, Cambridge, CB3 0WA, UK
}

\begin{abstract}
The $(2 k+2)$-dimensional Einstein-Yang-Mills equations for gauge group $S O(2 k)$ (or $S U(2)$ for $k=2$ and $S U(3)$ for $k=3$ ) are shown to admit a family of spherically-symmetric magnetic monopole solutions, for both zero and non-zero cosmological constant $\Lambda$, characterized by a mass $m$ and a magnetic-type charge. The $k=1$ case is the Reissner-Nordstrom black hole. The $k=2$ case yields a family of self-gravitating Yang monopoles. The asymptotic spacetime is Minkowski for $\Lambda=0$ and anti-de Sitter for $\Lambda<0$, but the total energy is infinite for $k>1$. In all cases, there is an event horizon when $m>m_{c}$, for some critical mass $m_{c}$, which is negative for $k>1$. The horizon is degenerate when $m=m_{c}$, and the near-horizon solution is then an $a d S_{2} \times S^{2 k}$ vacuum.
\end{abstract}

\footnotetext{
1 g.w.gibbons@damtp.cam.ac.uk

2 p.k.townsend@damtp.cam.ac.uk
} 


\section{Introduction}

In 1977 Yang found a spherically-symmetric solution of the 5-dimensional Euclidean $S U$ (2) Yang-Mills (YM) equations that provides a natural generalization of the Dirac magnetic monopole solution of Maxwell's equations in 3 space dimensions [1]. Just as the Dirac monopole is a point singularity in 3-space for which the integral of the magnetic 2-form is non-zero over any 2-sphere enclosing the singularity, so the Yang monopole is a point singularity in 5-space for which the integral of $\operatorname{tr}(\mathrm{F} \wedge \mathrm{F})$ is nonzero over any 4 -sphere enclosing the singularity, where $F$ is the $s u(2)$-valued YM 2 -form field-strength. Just as the Dirac monopole is effectively a homogeneous and isotropic Maxwell field strength on the 2-sphere, so the Yang monopole is effectively a homogeneous and isotropic $S U(2)$ instanton on the 4-sphere [2]. There are several important differences, however; for example, whereas Dirac's monopole charge can be any multiple of a fundamental unit, Yang's monopole charge has a fixed magnitude and only its sign can be freely chosen. In recent years, the Yang monopole has attracted the attention of condensed matter theorists because of its relevance to the 4-dimensional Quantum Hall Effect [3]. This application has led to the construction of some analogous higher-dimensional Yang-Monopole-type configurations [4, 5, 6, , 7 , 8. 9, which are effectively conformal maps to even-dimensional spheres of higherdimensional instantons [10, 11, 12].

Neither the Yang monopole nor its higher-dimensional analogs have yet had much impact on particle physics, presumably because of the infra-red divergent magnetic field energy, as against the ultra-violet divergent energy of a Dirac monopole. An ultra-violet divergence can be removed by a cut-off that has no effect on low-energy physics, but an infra-red divergence is more problematic. However, infinite-energy 'solitons' are now commonplace in string/M-theory studies because there is often a physical interpretation in terms of branes. In addition, it has recently been suggested that the endpoint of an $S O(32)$ heterotic string could be viewed as a kind of higherdimensional monopole [13. For the Yang monopole itself, the energy within a volume of radius $r$ diverges linearly with $r$, suggesting a possible interpretation as a 'splayed' string. In confirmation of this interpretation, we note that the YM flux would get confined to an instanton flux tube in a Higgs phase, with a Yang-type monopole as its endpoint, just as the magnetic flux of a Dirac magnetic monopole would be confined to a magnetic flux tube in a superconducting vacuum.

These considerations suggest that the Yang monopole, and its higher-dimensional analogs, deserve further study. One purpose of this paper is to present a construction of Yang-type monopoles in $\mathbb{E}^{2 k+1}$ for gauge group $S O(2 k)$. The construction is similar, but apparently not identical, to other constructions that have appeared previously in the physics literature; from a purely mathematical perspective, it has a straightforward generalization to homogeneous YM fields on homogeneous Einstein manifolds, but the physical interpretation of such 'generalized monopole' solutions remains to be explored. For $k=1$ the construction yields the Dirac monopole and for $k=2$ it yields a superposition of Yang monopoles in each of the two $S U(2)$ factors of $S O(4)$; one may consistently truncate to a Yang-monopole solution of the $S U(2) \mathrm{YM}$ equations. For $k=3$ one may consistently truncate to an $S U(3)$ subgroup of $S O(6)$. 
For $k=4$ the construction yields what could be called an "octonionic monopole" solution of the $S O(8)$ YM equations; this configuration was found in [6] but it was not appreciated there that it solves the YM equations.

All these solutions of the Euclidean YM equations can of course be viewed as static spherically-symmetric solutions of the YM equations on $(2 k+2)$-dimensional Minkowski spacetime, and another purpose of this paper is to obtain the corresponding static solutions of the coupled Einstein-YM equations. Our starting point for this purpose is the Lagrangian density

$$
\mathcal{L}=\sqrt{-\operatorname{det} g}\left[\frac{1}{16 \pi G}(R-2 \Lambda)-\frac{1}{2 \gamma^{2}} \operatorname{tr}|\mathrm{F}|^{2}\right],
$$

where $F$ is the field-strength for a YM theory with coupling constant $\gamma$, and $g$ is the metric of a $(2 k+2)$-dimensional spacetime, and $\Lambda$ is the cosmological constant. Our principal interest is in the $k>1$ cases, and we find a self-gravitating Yang monopole for $k=2$, and investigate its global geometry. The $k>2$ self-gravitating "Yang-type" monopoles are found to have similar properties. It turns out that spherical symmetry allows a family of self-gravitating solutions, parametrized by a mass $m$. For positive $m$ there is an event horizon that 'clothes' a spacelike singularity and for $\Lambda=0$ one can view the resulting solution as a 'Yang-monopole black hole' in the sense that the spacetime is asymptotically flat, albeit in a 'weak' sense, since the total energy is infinite. For $\Lambda<0$ the solution is asymptotically anti-de Sitter (adS) in the same sense.

For $\Lambda=0$ we present details of the global structure as a function of $m$. A peculiarity (for $k>1$ ) is that the absence of naked singularities is compatible with negative $m$, for which the singularity is timelike. Specifically, there is a negative 'critical' mass $m_{c}$ such that for $0>m>m_{c}$ there is both an inner (Cauchy) horizon and an outer (event) horizon; the global structure in this case is similar to that of the Reissner-Nordstrom (RN) black hole. When $m=m_{c}$, the two horizons coincide to form a degenerate event horizon, like that of the extreme RN black hole. Just as the near-horizon limit of the extreme RN black hole yields the Robinson-Bertotti $a d S_{2} \times S^{2}$ vacuum of the 4-dimensional Einstein-Maxwell theory, so the near-horizon limit of the extreme self-gravitating Yang-type monopole yields an $a d S_{2} \times S^{2 k}$ vacuum of the $(2 k+2)$-dimensional Einstein-YM theory. For $k=2$, this type of vacuum has been discussed previously under the rubric of "instanton-induced compactification" [14, but the $k>2$ cases yield Kaluza-Klein vacua that, to our knowledge, have not previously been considered. In any case, this type of vacuum may now be seen as a limit of a more general solution, just as many of the adS vacua of supergravity theories can be viewed as limits of brane spacetimes 15.

For $\Lambda<0$, the global structure is more complicated but similar for large adS radius. The spacetime is weakly asymptotically anti-de Sitter, because the metric tends to the adS metric but too slowly for the adS energy to be finite. Nevertheless, the adS boundary is an $S^{2 k} \times S^{1}$ spacetime, in which the $2 k$-sphere has a background $\operatorname{tr} \mathrm{F}^{\mathrm{k}}$ magnetic field (or a uniform Maxwell magnetic 2-form $F$ in the $k=1$ case). But this is precisely, the vacuum of a quantum Hall system, so it is natural to conjecture that quantum gravity in a weakly asymptotically adS background is dual to a 
noncommutative Quantum Hall theory. A boundary field theory at zero temperature would require a $(2 k+2)$-dimensional 'bulk' solution with a degenerate event horizon, but there always exists such a solution.

We should point out that self-gravitating Yang-type monopoles differ in essential respects from the 'monopole' solutions of the Einstein-YM equations found numerically by Bartnik and McKinnon [16, and extended to the asymptotically anti-de Sitter case and higher dimensions in [17. In our notation, this type of self-gravitating 'monopole' would be a solution of $S O(2 k+1)$ YM equations in a $(2 k+2)$-dimensional spacetime, for any integer $2 k$. These solutions are such that the field strength has components in the radial direction, as well as on the $2 k$-sphere. The absence of such radial components is presumably the difference that makes the self-gravitating Yang-type monopole equations tractable analytically.

\section{Preliminaries}

The Euler-Lagrange equations for the Lagrangian density (1.1) consist of the sourceless YM equations in a general $(2 k+2)$-dimensional spacetime, and Einstein equations for the metric of this spacetime with a YM source. The YM potential 1-form can be written in coordinates $\left\{x^{m} ; m=0,1, \ldots 2 k+1\right\}$ as

$$
A=d x^{m} A_{m}^{a} T_{a},
$$

where the matrices $T_{a}$ span a representation $R$ of the Lie algebra of the (semi-simple) gauge group $G$. If $f_{a b}^{c}$ are the structure constants of the algebra then

$$
\left[T_{a}, T_{b}\right]=i f_{a b}^{c} T_{c}
$$

We assume that $R$ is the fundamental representation and adopt the normalization convention

$$
2 \operatorname{tr}\left(\mathrm{T}_{\mathrm{a}} \mathrm{T}_{\mathrm{b}}\right)=\delta_{\mathrm{ab}}
$$

In particular, for $G=S U(2)$ we have $T_{a}=\sigma_{a} / 2$, where $\sigma_{a}(a=1,2,3)$ are the Pauli matrices. The YM equation is

$$
\partial_{m} \sqrt{-\operatorname{det} g} F^{m n}=i \sqrt{-\operatorname{det} g}\left[A_{m}, F^{m n}\right],
$$

The Einstein equation is

$$
G_{m n}=(8 \pi G) T_{m n}-g_{m n} \Lambda
$$

where the YM stress tensor is

$$
T_{m n}=\gamma^{-2}\left[\operatorname{tr}\left(\mathrm{F}_{\mathrm{m}}^{\mathrm{p}} \mathrm{F}_{\mathrm{np}}\right)-\frac{1}{4} \mathrm{~g}_{\mathrm{mn}} \operatorname{tr}\left(\mathrm{F}_{\mathrm{pq}} \mathrm{F}^{\mathrm{pq}}\right)\right] .
$$

For notational purposes it is convenient to consider the corresponding quadratic differential $T \equiv T_{m n} d x^{m} d x^{n}$. 
We seek spherically-symmetric solutions of these equations for which the spacetime metric takes the form

$$
d s^{2}=-\Delta d t^{2}+\Delta^{-1} d r^{2}+r^{2} d \Omega_{2 k}^{2}
$$

for some function $\Delta(r)$, where $d \Omega_{2 k}^{2}$ is the $S O(2 k-1)$-invariant metric on the unit $2 k$-sphere. These Schwarzschild-type coordinates are useful for many purposes, but it simpler to present the solution for the YM potential in terms of isotropic coordinates for which

$$
d s^{2}=-f d t^{2}+\omega^{2}\left[d \rho^{2}+\rho^{2} d \Omega_{2 k}^{2}\right]
$$

for functions

$$
f(\rho)=\Delta(r(\rho)), \quad \omega(\rho)=r(\rho) / \rho,
$$

where $r(\rho)$ is found by solving the differential equation

$$
\frac{d r}{r \sqrt{\Delta(r)}}=\frac{d \rho}{\rho} .
$$

We shall find it convenient to introduce coordinates $\left\{n^{i} ; i=1, \ldots, 2 k\right\}$ for $S^{2 k}$ such that

$$
d \Omega_{2 k}^{2}=h_{i j} d n^{i} d n^{j}
$$

where

$$
h_{i j}=\delta_{i j}+\frac{n^{i} n^{j}}{1-n^{2}}, \quad\left(n^{2}=\delta_{i j} n^{i} n^{j}\right) .
$$

Note that these coordinates cover only one hemi- $2 k$-sphere and are singular on the equatorial $(2 k-1)$-sphere at $n^{2}=1$. The volume form on $S^{2 k}$ in these coordinates is

$$
\operatorname{vol}\left(S^{2 k}\right)=\left(1-n^{2}\right)^{-\frac{1}{2}} d n^{1} \wedge \ldots \wedge d n^{2 k}
$$

\section{The Yang monopole}

We now review the Yang-monopole solution for $k=2$ in a way that will also allow preliminary consideration of the $k>2$ case, which we take up in the following section. The Yang monopole has 1-form potential

$$
A=\frac{\Sigma_{i j} n^{i} d n^{j}}{1+\sqrt{1-n^{2}}}
$$

where

$$
\Sigma_{i j}=\eta_{i j}^{a} T_{a}
$$

for constants $\eta_{i j}^{a}$ satisfying

$$
\eta_{i j}^{a} \eta_{k l}^{b} f_{a b}^{c}=2\left(\delta_{l[i} \eta_{j] k}^{c}-\delta_{k[i} \eta_{j] l}^{c}\right)
$$


Using this relation, it can be shown that the only non-vanishing components of $F$ are

$$
F_{i j}=\Sigma_{i j}-2 \frac{\delta_{l[i} \Sigma_{j] k} n^{l} n^{k}}{1-n^{2}+\sqrt{1-n^{2}}} .
$$

We also record here that

$$
F^{i j} \equiv \rho^{-4} h^{i k} h^{j l} F_{k l}=\rho^{-4}\left(\Sigma_{i j}+2 \frac{\delta_{l[i} \Sigma_{j] k}^{a} n^{l} n^{k}}{1+\sqrt{1-n^{2}}}\right) .
$$

Using (13.17) again, it may be verified that

$$
(\sqrt{\operatorname{det} h})^{-1} \partial_{i}\left(\sqrt{\operatorname{det} h} F^{i j}\right)=-i\left[A_{i}, F^{i j}\right]=\frac{2(k-1)}{\left(1+\sqrt{1-n^{2}}\right) \rho^{4}} n_{k} \Sigma_{j k},
$$

and hence that the YM equations are satisfied. This result is conditional on the existence of an constant " $\eta$-tensor" satisfying (3.17) but this is satisfied for $k=2$ and gauge group $S U(2)$ by the self-dual 't Hooft tensor (a quaternionic structure on $\mathbb{E}^{4}$ ), and this fact motivates the notation. Note that the anti-self-dual 't Hooft tensor $\bar{\eta}_{i j}^{a}$, also satisfies (3.17) and this yields a Yang anti-monopole. As shown by Yang [1], these are the only possibilities compatible with spherical symmetry (which is not manifest in the above formula for $F$ but which will be seen to be a property of all gauge-invariant quantities constructed from $F$ ).

It can be shown that

$$
\operatorname{tr} \mathrm{F}^{\mathrm{k}}=\left(1-\mathrm{n}^{2}\right)^{-\frac{1}{2}} \operatorname{tr} \Sigma^{\mathrm{k}} \quad\left(\Sigma=\frac{1}{2} \sum_{\mathrm{ij}} \mathrm{dn}^{\mathrm{i}} \mathrm{dn}^{\mathrm{j}}\right),
$$

where the exterior product of forms is implicit. It follows that $\operatorname{tr} \mathrm{F}^{\mathrm{k}}$ is proportional to the volume form on $S^{2 k}$. For $k=2$ we may use (2.4), and the 't Hooft tensor identities

$$
\eta_{[i j}^{a} \eta_{k l]}^{a} \equiv \varepsilon_{i j k l}, \quad \bar{\eta}_{[i j}^{a} \bar{\eta}_{k l]}^{a} \equiv-\varepsilon_{i j k l},
$$

to show that

$$
\pm \frac{1}{3} \operatorname{tr} \mathrm{F}^{2}=\left(1-\mathrm{n}^{2}\right)^{-\frac{1}{2}} \mathrm{dn}^{1} \wedge \mathrm{dn}^{2} \wedge \mathrm{dn}^{3} \wedge \mathrm{dn}^{4},
$$

which is the volume form on the unit 4-sphere. As the unit 4-sphere has 4-volume $8 \pi^{2} / 3$, we deduce that

$$
\frac{1}{8 \pi^{2}} \int_{S^{4}} \operatorname{tr} \mathrm{F} \wedge \mathrm{F}= \pm 1
$$

and hence that the Yang monopole configuration is equivalent to an instanton or anti-instanton on a 4 -sphere [2].

To conclude our discussion of the flat-space Yang monopole, we note that if

$$
\operatorname{tr}\left(\Sigma_{\mathrm{ik}} \Sigma_{\mathrm{kj}}\right)=-\frac{1}{2} \mathrm{~N} \delta_{\mathrm{ij}}
$$

where the constant $N$ must be such that

$$
\sum_{i<j} \operatorname{tr} \Sigma_{\mathrm{ij}} \Sigma_{\mathrm{ij}}=\frac{\mathrm{kN}}{2}
$$


then

$$
\operatorname{tr}\left(\mathrm{F}_{\mathrm{ik}} \mathrm{F}^{\mathrm{jk}}\right)=\frac{\mathrm{N}}{2 \rho^{4}} \delta_{\mathrm{i}}^{\mathrm{j}}
$$

The energy density is therefore

$$
\mathcal{E}(\rho) \equiv T_{00}=\frac{1}{4 \gamma^{2}} \operatorname{tr}\left(\mathrm{F}_{\mathrm{ij}} \mathrm{F}^{\mathrm{ij}}\right)=\frac{\mathrm{kN}}{4 \gamma^{2} \rho^{4}},
$$

which is spherically-symmetric. It follows (for $k>1$ ) that the total energy within a radius $R$ grows like $R^{2 k-3}$. For $k=2$, and $G=S U(2)$, the ' $\eta$-tensor' is the 't Hooft tensor and (3.25) is satisfied with $N=3$. The total energy of the Yang-monopole within a radius $R$ is therefore

$$
E(R)=\frac{8 \pi^{2}}{3} \int_{0}^{R} \mathcal{E} d \rho=(2 \pi / \gamma)^{2} R
$$

The coefficient $(2 \pi / \gamma)^{2}$ is precisely the tension of the instanton string, so the Yang monopole is marginally stable against collapse to an instanton flux string, although (as mentioned in the introduction) it will likely be unstable if the $S U(2)$ gauge symmetry is spontaneously broken.

\section{Higher-dimensional monopoles}

For gauge group $S O(2 k)$ the ' $\eta$-tensor' relation (3.17) is equivalent to the $s o(2 k)$ commutation relations

$$
\left[\Sigma_{i j}, \Sigma_{k l}\right]=2 i\left(\delta_{l[i} \Sigma_{j] k}-\delta_{k[i} \Sigma_{j] l}\right)
$$

The YM equations are therefore satisfied by $A$ of the form (3.15) provided that the $k(2 k-1)$ matrices $\Sigma_{i j}$ span the Lie algebra $s o(2 k)$. In this case the constants $\eta_{i j}^{a}$ just specify the linear transformation from the basis of $s o(2 k)$ provided by the matrices $T_{a}$ to the basis provided by the matrices $\Sigma_{i j}$. Let $\omega_{i j}$ be the components of $A$ in this new basis:

$$
A=\frac{1}{2} \omega_{i j} \Sigma_{i j}
$$

We can express these components in dyad form as

$$
\omega=\frac{\tilde{\mathbf{n}} d \mathbf{n}-d \tilde{\mathbf{n}} \mathbf{n}}{1+\sqrt{1-n^{2}}}
$$

where $\mathbf{n}$ is the column $2 k$-vector with components $n^{i}$, and $\tilde{\mathbf{n}}$ its transposed row vector. This $S O(2 k)$ gauge potential satisfies the YM equations on $S^{2 k}$ and hence the YM equations on $\mathbb{E}^{2 k+1}$. In fact, this applies not only for integer $k$ but also for half-integer $k$, in the sense that an $\mathrm{SO}(\mathrm{n})$ potential of the form (3.15) solves the YM equation on $\mathbb{E}^{n+1}$ for all $n \geq 2$.

This result has a simple geometrical interpretation, which we now present. Recall that the potential of a Dirac monopole is the connection on the principal $S O(2)$ 
bundle over a unit 2-sphere that has a 3 -sphere as the total space. The metric on this 3-sphere takes the form $(\sigma-\omega)^{2}+d \Omega_{2}^{2}$, where $\sigma=d \varphi$ is the invariant 1-form on the $S^{1}$ fibre, in its parametrization by the angle $\varphi$, and $d \Omega_{2}^{2}$ is the $S O(3)$-invariant metric on the 2 -sphere base. The $S O(2)$ connection $\omega$ needed such that the bundle metric is that of the unit 3 -sphere is precisely the $n=2(k=1)$ case of (4.32). In general, the potential (4.32) is the connection for the principal $S O(2 k)$ bundle over a unit $2 k$-sphere that has $S O(2 k+1)$ as its total space. To see this ${ }^{1}$, we express $g \in S O(n+1)$ as $g=h k$, where $h \in S O(n)$ and $k$ is a representative of the coset space $S O(n+1) / S O(n) \cong S^{n}$. The bi-invariant metric on $S O(n+1)$ is

$$
d l^{2}=\frac{1}{2} \operatorname{tr}\left(\mathrm{g}^{-1} \mathrm{dg}\right)^{2}=\frac{1}{2} \operatorname{tr}\left(\mathrm{h}^{-1} \mathrm{dh}+\mathrm{dkk}^{-1}\right)^{2},
$$

where the trace is over the $(n+1) \times(n+1)$ matrices of the vector representation. To express this metric in the required form, we need expressions for the left-invariant forms $h^{-1} d h$ on $S O(n)$ and the right-invariant forms $d k k^{-1}$ on $S^{n}$. For the obvious embedding of $S O(n)$ in $S O(n+1)$ we have

$$
h^{-1} d h=\left(\begin{array}{ll}
\sigma & 0 \\
0 & 0
\end{array}\right)
$$

for left-invariant forms $\sigma$ on $S O(n)$. For $k$ we may choose

$$
k=\exp \left(\begin{array}{cc}
0 & \mathbf{b} \\
-\tilde{\mathbf{b}} & 0
\end{array}\right)=\left(\begin{array}{cc}
1-\frac{\mathbf{n} \tilde{\mathbf{n}}}{1+\sqrt{1-n^{2}}} & \mathbf{n} \\
-\tilde{\mathbf{n}} & \sqrt{1-n^{2}}
\end{array}\right),
$$

where

$$
\mathbf{n}=(\sin |\mathbf{b}| /|\mathbf{b}|) \mathbf{b}
$$

A calculation then shows that

$$
k^{-1} d k=\left(\begin{array}{cc}
\omega & \mathbf{v} \\
-\tilde{\mathbf{v}} & 0
\end{array}\right), \quad d k k^{-1}=\left(\begin{array}{cc}
-\omega & \mathbf{v} \\
-\tilde{\mathbf{v}} & 0
\end{array}\right)
$$

where $\omega$ is precisely the connection of (4.32), and

$$
\mathbf{v}=d \mathbf{n}+\mathbf{n} \frac{(\tilde{\mathbf{n}} d \mathbf{n})}{1-n^{2}+\sqrt{1-n^{2}}} .
$$

Thus,

$$
h^{-1} d h+d k k^{-1}=\left(\begin{array}{cc}
\sigma-\omega & \mathbf{v} \\
-\tilde{\mathbf{v}} & 0
\end{array}\right),
$$

and hence the bi-invariant metric on $S O(n+1)$ is

$$
d l^{2}=\frac{1}{2} \operatorname{tr}(\sigma-\omega)^{2}+\tilde{\mathbf{v}} \mathbf{v},
$$

\footnotetext{
${ }^{1}$ Here we follow Appendix B of [18], where a Kaluza-Klein interpretation can be found.
} 
where the trace is now over the $n \times n$ matrices of the vector representation of $S O(n)$. One finds that

$$
\tilde{\mathbf{v}} \mathbf{v}=d \tilde{\mathbf{n}}\left(1-\frac{\mathbf{n} \tilde{\mathbf{n}}}{1-n^{2}}\right) d \mathbf{n}
$$

which is the metric on the unit $n$-sphere.

Although this construction applies for any $n$, we must distinguish between even $n$ and odd $n$. For $n=2 k$ with integer $k$ the YM configuration on $S^{2 k}$ is topologically non-trivial because the integral of $\operatorname{tr} \mathrm{F}^{\mathrm{k}}$ over the $2 k$-sphere is non-zero. This integral is a conserved magnetic charge that justifies the 'monopole' terminology. As we have seen, the $k=1$ case yields the Dirac monopole. The $k=2$ case yields a solution of the $S O(4)$ YM equations that is a superposition of Yang monopoles in each of the two $S U(2)$ factors of $S O(4)$. For $k=4$ we have an "octonionic monopole" solution of the $S O(8)$ YM equations. In contrast, the odd-sphere cases are better thought of as $S O(n+1)$-invariant instantons on $\mathbb{E}^{n+1}$. For $n=3$, the construction yields an $S O$ (4)-invariant configuration of $S O(3)$ YM fields on the 3-sphere, but this is just the spherically-symmetric 1-instanton solution on $\mathbb{E}^{4}$. Similarly, for $n=5$ we find an $S O(6)$-invariant instanton of the $S O(5)$ YM equations on $\mathbb{E}^{6}$, and for $n=7$ an $S O(8)$-invariant instanton of the $S O(7)$ YM equations on $\mathbb{E}^{8}$. The latter is presumably related to the "octonionic instanton" of [19, 20].

\section{$5 \quad$ Self-gravitating Yang-type monopoles}

Because a Yang-type monopole has a field-strength 2-form with components only on the $2 k$-sphere, it can be viewed as a static solution in any $(2 k+2)$-dimensional spacetime with metric of the form (2.9). However, we then need to solve the Einstein equation for the YM source to determine the functions appearing in this metric ansatz. A Yang-type monopole has the spherically-symmetric (quadratic differential) stress tensor

$$
T=\frac{k N}{4 \gamma^{2} \omega^{4} \rho^{4}}\left[f d t^{2}+\omega^{2} d \rho^{2}-[(k-2) / k] \omega^{2} \rho^{2} d \Omega_{2 k}^{2}\right],
$$

where $N$ is the constant appearing in (3.25). In the Schwarzschild-type coordinates, for which the spacetime metric is (2.8), we have

$$
T=\frac{k N}{4 \gamma^{2} r^{4}}\left[\Delta d t^{2}+\Delta^{-1} d r^{2}-[(k-2) / k] r^{2} d \Omega_{2 k}^{2}\right] .
$$

If we write

$$
\Delta(r)=1-\frac{2 G M(r)}{r^{2 k-1}}
$$

for "mass function" $M(r)$, then the Einstein equations reduce, for $\Lambda=0$, to the ordinary differential equation ${ }^{2}$

$$
\frac{d M}{d r}=\frac{N \pi}{\gamma^{2}} r^{2(k-2)}+\frac{\Lambda}{2 k G} r^{2 k} .
$$

\footnotetext{
${ }^{2}$ See, for example, section 6 of [21].
} 
The solution is ${ }^{3}$

$$
M(r)=m+\frac{N \pi}{(2 k-3) \gamma^{2}} r^{2 k-3}+\frac{(\Lambda / G)}{2 k(2 k+1)} r^{2 k+1},
$$

for arbitrary constant $m$, which has dimensions of mass. This yields

$$
\Delta=1-\frac{2 G m}{r^{2 k-1}}-\frac{\mu^{2}}{r^{2}}-\frac{\Lambda r^{2}}{k(2 k+1)}
$$

where

$$
\mu^{2}=\frac{2 \pi G N}{(2 k-3) \gamma^{2}}
$$

Note that $\mu^{2}<0$ for $k=1$, in which case we should set $N=1$; for $\Lambda=0$ we then have the Reissner-Nordstrom metric. We now consider in turn the subcases of zero, negative and positive $\Lambda$.

\section{$5.1 \Lambda=0$}

For $\Lambda=0$, the spacetime metric is

$$
d s^{2}=-\frac{P(r)}{r^{2 k-1}} d t^{2}+\frac{r^{2 k-1}}{P(r)} d r^{2}+r^{2} d \Omega_{2 k}^{2},
$$

where $P(r)$ is the polynomial

$$
P(r)=r^{2 k-1}-\mu^{2} r^{2 k-3}-2 G m .
$$

When $m=0$ we have a 'pure' self-gravitating Yang-type monopole with spacetime metric

$$
d s^{2}=-\left(1-\frac{\mu^{2}}{r^{2}}\right) d t^{2}+\left(1-\frac{\mu^{2}}{r^{2}}\right)^{-1} d r^{2}+r^{2} d \Omega_{2 k}^{2} .
$$

There is an event horizon at $r=\mu$ behind which we find a spacelike singularity at $r=0$. This is therefore a kind of Yang-monopole black hole. Integration of (2.11) with $r H(r)=\sqrt{r^{2}-\mu^{2}}$ yields

$$
r=\frac{\mu}{2}\left(\frac{\rho}{\rho_{0}}+\frac{\rho_{0}}{\rho}\right)
$$

for arbitrary constant $\rho_{0}$. We see that isotropic coordinates cover only the region outside the horizon, as expected, but also that there are two isometric exterior regions, with $\rho>\rho_{0}$ and $\rho<\rho_{0}$. These regions are connected on a hypersurface of constant $t$ by a 'throat' with minimum $2 k$-sphere of radius $\mu$ at $\rho=\rho_{0}$. The $\operatorname{tr} \mathrm{F}^{\mathrm{k}}$ flux of the monopole passes through this throat. Note that in the region $\rho<\rho_{0}$, the singularity of the YM field at $\rho=0$ corresponds to a source of the flux at $r=\infty$.

\footnotetext{
${ }^{3}$ This also applies for half-integer $k$, with the exception of $k=3 / 2$. The $k=3 / 2$ case was analysed in [22].
} 
For 'impure' solutions with $m \neq 0$ the horizon structure may be more complex. Note that the polynomial $P(r)$ has a minimum at positive $r$ and a maximum at negative $r$, and no other extrema. It follows that $P$ has at most three real roots. Let us consider how these roots change with $m$, starting with $m=0$, where we have one real postive root, one real negative root and one root at $r=0$. As $m$ increases from zero, the root at $r=0$ moves to negative $r$, where it must either stay as $m$ continues to increase monotonically (since a root can cross the $r=0$ axis only at $m=0$ ) or disappear (by coalescence with the other negative root, which must also stay negative). Thus, there is a unique real positive root $r=r_{+}$for $m>0$, corresponding to a non-degenerate event horizon behind which there is a spacelike singularity at $r=0$. Moreover, $r_{+}$increases monotonically with $m$, so that matter falling into the 'black hole' increases the surface area of the horizon, as one would expect.

However, $m$ need not be positive. As we decrease $m$ from zero, the zero root of $P(r)$ now moves to positive $r$, so we now have two real positive zeros of $P(r)$, corresponding to an inner (Cauchy) horizon at $r=r_{-}$and an outer (event) horizon at $r=r_{+}>r_{-}$. As before, this state of affairs cannot change as $m$ continues to decrease monotonically to ever larger negative values until the two roots $r=r_{ \pm}$ coincide, after which there is no longer any real positive root and hence no event horizon. Thus, there exists a 'critical' negative value $m_{c}$ of $m$, such that there is an event horizon for $m>m_{c}$ but a naked (timelike) singularity for $m<m_{c}$. In fact,

$$
G m_{c}=-\frac{1}{2 k-3}\left(\frac{2 \pi G N}{(2 k-1) \gamma^{2}}\right)^{k-\frac{1}{2}} .
$$

For $m_{c}<m<0$ the global structure is similar to that of the Reissner-Nordstrom black hole. When $m=m_{c}$, the inner and outer horizons coalesce to form a degenerate (zero surface gravity) event horizon. The global geometry is then similar to that of the extreme Reissner-Nordstrom spacetime, and the near-horizon limit of this 'extremal' self-gravitating Yang-type monopole is an $a d S_{2} \times S^{2 k}$ vacuum solution.

To illustrate these general observations, we will consider the $k=2$ (Yang-monopole) case in more detail. In this case the polynomial $P(r)$ is cubic,

$$
P(r)=r^{3}-\mu^{2} r-2 G m,
$$

and there are no naked singularities as long as $3 \sqrt{3} \mathrm{Gm} / \mu^{3} \geq-1$, or

$$
m \gamma^{3} / \sqrt{G} \geq-(2 \pi)^{\frac{3}{2}} .
$$

At saturation, the cubic polynomial is

$$
P(r)=\left(r+\frac{2 \mu}{\sqrt{3}}\right)\left(r-\frac{\mu}{\sqrt{3}}\right)^{2} .
$$

The double zero at $r=\mu / \sqrt{3}$ means that the inner and outer horizons have now merged to form a single degenerate horizon at $r=\mu / \sqrt{3}$. Since

$$
\frac{d \rho}{\rho}=\frac{d r}{\left(r-\frac{\mu}{\sqrt{3}}\right) \sqrt{1+\frac{2 \mu}{\sqrt{3} r}}},
$$


we have, near the horizon at $r=\mu / \sqrt{3}$,

$$
\rho^{\sqrt{3}} \sim\left(r-\frac{\mu}{\sqrt{3}}\right)
$$

so that $\rho \rightarrow 0$ at the horizon. Introducing the new time variable

$$
\lambda=\frac{3 \sqrt{3}}{\mu^{2}} t
$$

we may write the near horizon metric as

$$
d s^{2}=\frac{\mu^{2}}{3}\left[-\rho^{2 \sqrt{3}} d \lambda^{2}+\left(\frac{d \rho}{\rho}\right)^{2}+d \Omega_{4}^{2}\right] .
$$

This is a metric for $a d S_{2} \times S^{4}$, and the singularity at $\rho=0$ is a coordinate singularity at the adS horizon.

\section{$5.2 \Lambda<0$}

For a 'pure' $m=0$ monopole, we have

$$
\Delta=1-\frac{\mu^{2}}{r^{2}}+\frac{r^{2}}{\ell^{2}}
$$

for $\Lambda<0$, where

$$
\ell=\sqrt{k(2 k+1)|/| \Lambda \mid}
$$

is the adS radius. There is a unique non-degenerate horizon at

$$
r=\frac{1}{\sqrt{2}} \sqrt{\sqrt{\ell^{4}+4 \mu^{2} \ell^{2}}-\ell^{2}} .
$$

For simplicity, we shall concentrate for $m \neq 0$ on the $k=2$, Yang-monopole, case, for which

$$
\Delta=1-\frac{2 G m}{r^{3}}-\frac{\mu^{2}}{r^{2}}+\frac{r^{2}}{\ell^{2}} .
$$

The horizon structure is qualitatively similar for higher $k$. For positive $m$ there is still a unique non-degenerate event horizon. For negative $m$ there is a critical mass $m_{c}$ such that there exists both an inner and outer horizon for $0>m>m_{c}$, with a unique degenerate horizon when $m=m_{c}$, and a naked singularity when $m<m_{c}$. For $k=2$ we have

$$
m_{c}=-r_{c}^{2}\left(1+2 r_{c}^{2} / \ell^{2}\right)
$$

where $r_{c}$ is the position of the degenerate horizon, given by

$$
10 r_{c}^{2}=\ell\left(\sqrt{9 \ell^{2}+20 \mu^{2}}-3 \ell\right) .
$$

The global structure is thus similar to the $\Lambda=0$ case apart from the asymptotic behaviour for large $r$. 


\section{$5.3 \Lambda>0$}

For a 'pure' $m=0$ monopole we now have

$$
\Delta=1-\frac{\mu^{2}}{r^{2}}-\frac{r^{2}}{R^{2}}
$$

where

$$
R=\sqrt{k(2 k+1) \mid / \Lambda}
$$

is the de-Sitter radius (the radius of the minimal $2 k$-sphere). Now there is both an inner (event) and outer (cosmological) horizon for $R>2 \mu$. These horizons coalesce at $R=2 \mu$, and for $R<2 \mu$ there is a naked singularity at $r=0$.

For $m \neq 0$ we shall again concentrate on the $k=2$ case, for which

$$
\Delta=1-\frac{2 G m}{r^{3}}-\frac{\mu^{2}}{r^{2}}-\frac{r^{2}}{R^{2}}
$$

Let us consider increasing $m$ monotonically from zero. If there were initially two zeros of $\Delta$ then this will continue to be the case up to a maximum value of $m$, after which there is only a naked singularity. For $m<0$ there may be three horizons, an inner (Cauchy) horizon, an outer (event) horizon and a cosmological horizon, provided that

$$
R>R_{c} \equiv \frac{2 \sqrt{5}}{3} \mu
$$

and in this case there will be some negative $m$ for which the inner and outer horizons coalesce, yielding just two horizons, one a degenerate event horizon and the other the cosmological horizon. For $R \leq R_{c}$ there is only a cosmological horizon.

\section{Discussion}

We have shown that the interpretation of $S O(2 k+1)$ as an $S O(2 k)$ bundle over $S^{2 k}$ leads to a non-singular $S O(2 k+1)$-invariant configuration of $S O(2 k)$ Yang-Mills fields on $S^{2 k}$ with topological charge proportional to the integral of $\operatorname{tr} \mathrm{F}^{\mathrm{k}}$ over the $2 k$ sphere. Viewed as a singular configuration on $\mathbb{E}^{2 k+1}$, these are magnetic monopoles: $k=1$ yields the Dirac monopole and $k=2$ the Yang monopole; more precisely, a superposition of two Yang monopoles that is trivially truncated to a single Yang monopole. The $k>2$ cases yield higher-dimensional magnetic monopoles, such as a $k=4$ "octonionic" monopole.

The "Yang-type" monopoles can be viewed as static spherically-symmetric solutions of the $S O(2 k)$ YM equations on $(2 k+2)$-dimensional Minkowski space. We have extended these solutions of the flat-space YM equations to a class of static spherically-symmetric solutions of the $(2 k+2)$-dimensional Einstein-Yang-Mills equations, parametrized by a mass $m$, for arbitrary cosmological constant $\Lambda$.

When $\Lambda=0$, these solutions generalise the magnetic Reissner-Nordstrom black hole, which is the $k=1$ case. The $k=2$ case is a self-gravitating version of the 
Yang magnetic monopole, and there is an analogous solution for all higher $k$. All $k>1$ solutions have infinite total energy, the energy within a radius $r$ diverging as $r^{2 k-3}$. In all cases, there is an event horizon for $m>m_{c}$ where the 'critical' value $m_{c}$ of $m$ is negative (for $k \geq 2$ ). For $m_{c}<m<0$ the global structure is similar to that of the Reissner-Nordstrom black hole, and the $m=m_{c}$ solution is similar to the extreme Reissner-Nordstrom black hole. Specifically, the horizon is degenerate when $m=m_{c}$ and the near-horizon solution is an $a d S_{2} \times S^{2 k}$ vacuum. For $k=1$ this is just the Robinson-Bertotti solution of the Einstein-Maxwell equations and for $k=2$ it yields the solutions found previously by "instanton-induced compactification" [14]. The $k>2$ cases yield new Einstein-YM vacua.

The similarity of the extremal self-gravitating monopoles to the extreme ReissnerNordstrom black hole suggests a connection with supersymmetry. However, the total mass of either the Yang monopole or its higher-dimensional analogs is undefined, so it is hard to see how any well-defined charges given by integrals at spatial infinity could span a supersymmetry algebra. It is also difficult to see how Yang-type monopole solutions could be extended to solutions of supergravity theories. For example, 6dimensional supergravity-YM theories have a 3 -form field strength $H$ that obeys an 'anomalous' Bianchi identity of the form $d H \propto \operatorname{trF}^{2}$. It follows that $H$ cannot vanish in a Yang monopole background, but no non-zero $H$ could be compatible with spherical symmetry.

For $\Lambda<0$ one finds analogous 'monopole black hole' solutions for which the spacetime metric is weakly asymptotically adS . It is natural to suppose that the adS boundary is the vacuum for some holographically dual field theory, which must be a non-commutative Quantum Hall type theory as a consequence of the 'magnetic' flux through the $2 k$-sphere of the $S^{2 k} \times S^{1}$ boundary. Thus, the results presented here potentially provide a new way to study non-commutative field theories as holographic duals of gravitational theories.

Another application of our results is to wormhole solutions of the Euclidean Einstein-YM equations on $\mathbb{R} \times S^{n}$. For $\Lambda=0$, the metric is

$$
d s^{2}=d \tau^{2}+a^{2}(\tau) d \Omega_{n}^{2}
$$

where $a^{2}(\tau)=\tau^{2}+r_{0}^{2}$ for constant $r_{0}$. One may also consider Euclidean solutions of this type for $\Lambda \neq 0$. The construction for general $n$ is a straightforward extension of the $n=3$ case discussed in [23].

We conclude with a comment on the $k=3$ case. Our construction of higherdimensional monopoles rests on the interpretation of $S^{2 k}$ as the homogeneous space $S O(2 k+1) / S O(2 k)$, which is unique (at least locally) for all integer $k$ except $k=3$. The group $G_{2}$ acts transitively on $S^{6}$, leaving invariant the round metric (see e.g. [24]). The stabilizer is $S U(3)$, so

$$
S^{6} \cong G_{2} / S U(3)
$$

and this implies the existence of a $G_{2}$-invariant monopole solution of the $S U(3)$ YM equations on $\mathbb{E}^{7}$. The $G_{2}$-symmetry of this solution is compatible with non-zero $H$, and so a self-gravitating version of it might be extendible to a membrane-monopole of 
the heterotic string (not to be confused with the "octonionic membrane" of [25, 26], which is a solution of the equations of 10-dimensional supergravity coupled to a YM supermultiplet with $G_{2}$ gauge group). We leave this to a future investigation.

Acknowledgements: PKT is supported by a Senior Research Fellowship of the EPSRC. We thank Eric Bergshoeff and Harvey Reall for discussions.

\section{References}

[1] C. N. Yang, Generalization Of Dirac's Monopole To SU(2) Gauge Fields, J. Math. Phys. 19, 320 (1978).

[2] R. Jackiw and C. Rebbi, Conformal Properties Of A Yang-Mills Pseudoparticle, Phys. Rev. D 14, 517 (1976).

[3] S. C. Zhang and J. p. Hu, A Four Dimensional Generalization of the Quantum Hall Effect, Science 294 (2001) 823 arXiv:cond-mat/0110572.

[4] B. Chen, H. Itoyama and H. Kihara, Nonabelian monopoles from matrices: Seeds of the spacetime structure, Nucl. Phys. B 577, 23 (2000) arXiv:hep-th/9909075.

[5] M. Fabinger, Higher-dimensional quantum Hall effect in string theory, JHEP 0205 (2002) 037 arXiv:hep-th/0201016.

[6] B. A. Bernevig, J. p. Hu, N. Toumbas and S. C. Zhang, The Eight Dimensional Quantum Hall Effect and the Octonions, Phys. Rev. Lett. 91, 236803 (2003) arXiv:cond-mat/0306045.

[7] G. w. Meng, Geometric Construction of the Quantum Hall Effect in Higher Dimensions, J. Phys. A 36 (2003) 9415 arXiv:cond-mat/0306351;

[8] K. Hasebe and Y. Kimura, Dimensional hierarchy in quantum Hall effects on fuzzy spheres, Phys. Lett. B 602 (2004) 255 arXiv:hep-th/0310274.

[9] G. w. Meng, Dirac and Yang monopoles revisited, arXiv:math-ph/0409051.

[10] B. Grossman, T. W. Kephart and J. D. Stasheff, Solutions To Yang-Mills Field Equations In Eight-Dimensions And The Last Hopf Map, Commun. Math. Phys. 96 (1984) 431 [Erratum-ibid. 100 (1985) 311].

[11] D. H. Tchrakian, N-Dimensional Instantons And Monopoles, J. Math. Phys. 21, $166(1980)$.

[12] C. Saclioglu, Scale Invariant Gauge Theories And Selfduality In Higher Dimensions, Nucl. Phys. B 277, 487 (1986).

[13] J. Polchinski, Open heterotic strings, arXiv:hep-th/0510033. 
[14] S. Randjbar-Daemi, A. Salam and J. A. Strathdee, Instanton Induced Compactification And Fermion Chirality, Phys. Lett. B 132 (1983) 56.

[15] G. W. Gibbons and P. K. Townsend, Vacuum interpolation in supergravity via super p-branes, Phys. Rev. Lett. 71 (1993) 3754 arXiv:hep-th/9307049.

[16] R. Bartnik and J. Mckinnon, Particle - Like Solutions Of The Einstein YangMills Equations, Phys. Rev. Lett. 61, 141 (1988).

[17] E. Radu and D. H. Tchrakian, No hair conjecture, nonabelian hierarchies and anti-de Sitter spacetime, Phys. Rev. D 73, 024006 (2006) arXiv:gr-qc/0508033.

[18] M. Cvetic, G. W. Gibbons, H. Lu and C. N. Pope, Consistent group and coset reductions of the bosonic string, Class. Quant. Grav. 20 (2003) 5161 arXiv:hep-th/0306043.

[19] D. B. Fairlie and J. Nuyts, Spherically Symmetric Solutions Of Gauge Theories In Eight-Dimensions, J. Phys. A 17, 2867 (1984).

[20] S. Fubini and H. Nicolai, The Octonionic Instanton, Phys. Lett. B 155 (1985) 369.

[21] G. W. Gibbons, M. J. Perry and C. N. Pope, Bulk/boundary thermodynamic equivalence, and the Bekenstein and cosmic-censorship bounds for rotating charged AdS black holes, Phys. Rev. D 72, 084028 (2005) arXiv:hep-th/0506233.

[22] N. Okuyama and K. i. Maeda, Five-dimensional black hole and particle solution with non-Abelian gauge field, Phys. Rev. D 67, 104012 (2003) arXiv:gr-qc/0212022.

[23] A. Hosoya and W. Ogura, Wormhole Instanton Solution In The Einstein YangMills System, Phys. Lett. B 225, 117 (1989).

[24] A. J. Macfarlane, The sphere S(6) viewed as a $G(2) / S U(3)$ coset space, Int. J. Mod. Phys. A 17, 2595 (2002).

[25] T. A. Ivanova, Octonions, selfduality and strings, Phys. Lett. B 315, 277 (1993).

[26] M. Günaydin and H. Nicolai, Seven-dimensional octonionic Yang-Mills instanton and its extension to an heterotic string soliton, Phys. Lett. B 351 (1995) 169 [Addendum-ibid. B 376 (1996) 329] arXiv:hep-th/9502009. 